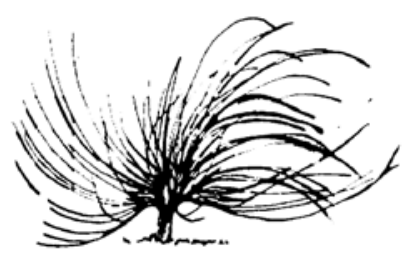

\title{
La enseñanza invertida (EI) o flipped teaching (FT) como método de mediación pedagógica aplicado a un curso de inglés integrado para otras especialidades en la Universidad Nacional (UNA), Costa Rica
}

\author{
Damaris Cordero Badilla ${ }^{1}$ \\ Universidad Nacional, Costa Rica, \\ Heredia, Costa Rica \\ jodaro.damaris@gmail.com \\ Vianey Martín Núñez Arguedas ${ }^{2}$ \\ Universidad Nacional, Costa Rica \\ Heredia, Costa Rica \\ martvna@yahoo.com
}

\section{Resumen}

Este artículo resume los resultados de un estudio de caso que tuvo como objetivo describir lo que sucede con la

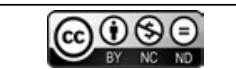

http://dx.doi.org/10.15359/rep.12-1.5

Recibido: 11 de marzo de 2016-Aprobado: 9 de mayo de 2017

1 Máster en Administración Universitaria (M.A.U.) de la Universidad de Costa Rica y Máster en Educación con énfasis en la Enseñanza del Inglés de la Universidad Latina de Costa Rica. Labora como docente en la Escuela de Literatura y Ciencias del Lenguaje de la UNA. Ha realizado trabajos de investigación en gramática comunicativa, estrategias metodológicas innovadoras y los usos de las TIC en la docencia universitaria, entre otros. Ha publicado artículos sobre estos temas en varias revistas nacionales e internacionales.

2 Máster en Lingüística Aplicada con Énfasis en Alumnado Adulto de la Universidad Nacional en Costa Rica, ha trabajado para el Ministerio de Educación Pública como profesor en diferentes colegios del país y desde el año 2006 labora en la Escuela de Literatura y Ciencias del Lenguaje de la Universidad Nacional en la enseñanza de distintas áreas de la lengua extranjera como cultura, gramática, conversación y otros. Actualmente se desempeña como el coordinador académico de los programas de idiomas extranjeros para otras carreras en la UNA. 
implementación del modelo de enseñanza llamado enseñanza invertida (EI)/ "flipped teaching (FT)" a un grupo de estudiantes de nivel pre-intermedio en un Curso de Inglés Integrado para otras carreras en la Universidad Nacional, Costa Rica. El estudio alude a las actividades de mediación pedagógica que se realizan, la labor llevada a cabo por su docente a cargo y el rendimiento de estudiantes meta durante 120 horas lectivas distribuidas en 17 semanas de estudio. Esta es una investigación que tomó como base la motivación e interés del alumnado hacia el aprendizaje de una lengua extranjera con la incorporación y uso de tecnologías y de medios de comunicación social. La información se recolectó a través de la puesta en práctica de un examen de diagnóstico, observaciones de clase y entrevistas hechas a estudiantes con la puesta en práctica de varios de los principios en los que se basa esta metodología de enseñanza. En este artículo se describen la metodología seguida, los problemas encontrados, y los logros alcanzados durante este periodo. Se analizará la información y los resultados obtenidos y se establecerán una serie de conclusiones generales sobre la pertinencia y el impacto de la metodología.

Palabras clave: enseñanza invertida (EI), enfoques la enseñanza del inglés, prácticas docentes, estudio de caso, mediación pedagógica.

\begin{abstract}
This paper summarizes the results of a case study directed to describe what actually happens with the implementation of the Flipped Teaching Approach in a group of intermediate students of English at the English for Other Majors Program at Universidad Nacional, Costa Rica. The study provides some insights into those activities used as pedagogical mediation tools within the English classroom, the teacher's role and the students' academic performance in a 120-hour program which lasts 17 weeks. This investigation considers students' interest and motivation towards the learning of a foreign language and the incorporation of new technologies and the usage of social media tools. The information was
\end{abstract}


collected through the use of a diagnostic exam, classroom observations, and interviews to the students, with the implementation of several of the principles on which this teaching methodology is based. This article describes the methodology followed, problems encountered, and achievements during this period. The information and results obtained will be analyzed and then, some general conclusions regarding pertinence and impact will be drawn.

Keywords: Flipped Teaching (FT), English Teaching approaches, teaching practices, case study, pedagogical mediation

\section{La buena didáctica es aquella que deja que el pensamiento del otro no se interrumpa y que le permite, sin notarlo, ir tomando buena dirección.}

Enrique Tierno Galván (1918-1986). Político e intelectual español.

\section{Introducción}

$\mathrm{E}$ 1 término "flipped classroom" fue creado por Bergmann y Sams en el año 2012, dos profesores de química de la Woodland Park High School, en Colorado, quienes impulsaron el uso de la videoconferencia como apoyo a alumnado que se ausentaba de sus salones de clase por alguna razón. De este modo, la enseñanza invertida es una forma de enseñanza y aprendizaje que permite al sujeto dicente acceder de forma remota a nuevos contenidos educativos con el uso de sesiones académicas pregrabadas, y luego serán asesoradas mediante actividades específicas de mediación pedagógica en el aula, en lugar de las tradicionales asignaciones extra clase o tareas (Santiago,2005, p.1).

Según indica Santiago (2015), el uso de este tipo de enseñanza, que es conocido como aula o instrucción invertida, aporta beneficios tanto para aprendientes como para docentes. Al estudiantado, porque le brinda la oportunidad de utilizar la información en el momento, lugar o tiempo deseado, de empoderarse de su aprendizaje, y de fortalecer sus competencias y habilidades de pensamiento crítico; y al cuerpo docente, de poder dedicar más tiempo a la atención de la diversidad en la 
clase, a la práctica, la aplicación de conceptos, y a la interacción con sus estudiantes y con colegas del área.

En una clase tradicional, cada estudiante trata de entender lo que su docente quiere enseñarle en un tiempo real, lo cual brinda pocas oportunidades para la reflexión, pero con la incorporación del video y otros medios previamente grabados, las clases quedan en manos de quienes aprenden, de modo que individualmente pueden reproducir, retroceder, adelantar o pausar la información de acuerdo con sus necesidades. Esta característica particular del material utilizado puede "resultar altamente beneficiosa para estudiantes quienes lidien con algún problema de accesibilidad a la información, según también detalla el portal mencionado" (EDUCASE.EDU, 2012, p. 1).

No obstante, Tourón (2013) menciona que, para que esta metodología sea efectiva, quien enseña de forma invertida necesita estar constantemente modificando y tratando de mejorar las experiencia según lo que resulte más efectivo para sus estudiantes. La utilización de un enfoque integral como este, que incluye una instrucción directa, y el uso de actividades de investigación, práctica y evaluación permite además a personal académico y dicente reflexionar sobre los procesos involucrados, y mejorar la calidad de lo que hacen en el salón de clase.

\section{La instrucción inversa en el aprendizaje del inglés como lengua extranjera}

Extendiendo los conceptos anteriores al aprendizaje de una lengua extranjera, la aplicación de la enseñanza invertida permite al personal académico asignar la teoría y los contenidos propios de cualquier programa para su estudio de forma remota, y su aplicación y práctica en el aula, facilitándole la preparación de actividades mejor enfocadas a los objetivos del curso. Por otro lado, permite al sujeto aprendiente revisar los contenidos gramaticales, léxicos y sintácticos propios de la lengua estudiada de forma que pueda llegar a la clase con algún conocimiento previo. Así podrá aclarar posibles dudas y maximizar su tiempo de producción en la lengua meta.

Bretzmann et al. (2013) hacen ver cómo una clase de inglés invertida es diferente a cualquier otra que utiliza este tipo de enfoque debido a los estándares ingleses, (especialmente los del Marco Común Europeo), por lo que es importante que el profesorado al implementarla 
debe pensar en ella como "una oportunidad de aprendizaje diferente para el estudiantado" (p. 229).

Generalmente, los procesos de enseñanza, aprendizaje y evaluación del inglés como lengua extranjera en las aulas del sistema educativo costarricense se realizan de la misma manera tanto en instituciones públicas como privadas, de educación básica, intermedia o superior; a saber; la presentación de la teoría y contenidos por parte del profesor, la práctica guiada en clase y la producción.

Así, la forma en que estas tres etapas han sido diseñadas deja lamentablemente a quien aprende con poco tiempo para producir, pues generalmente la etapa de presentación se extiende por varios minutos según cátedra dictada por la persona responsable del curso, la etapa de práctica se limita a una serie de ejercicios repetitivos que lejos de empoderar al estudiantado en su nuevo conocimiento lo encasilla en el uso irreal del idioma y, como se dijo anteriormente, le deja con muy poco tiempo de instrucción útil para la producción y utilización del idioma meta en actividades y situaciones prácticas que simulen su uso en la vida real. Generalmente, la producción en este tipo de diseño se asigna como trabajo para el hogar. Este diseño tradicional se ilustra en la Figura 1.

No obstante, el tema recurrente en la mayoría de las aulas de idioma extranjero es lo limitado que es el tiempo para cubrir estas tres etapas del proceso, al cual habrá que adicionarle las horas necesarias para evaluación y lo que da como resultado un evidente desfase entre los objetivos propuestos y los objetivos logrados.

Por otro lado, mucho se ha hablado de la importancia que han tomado las tecnologías de información y comunicación (TIC) en los procesos educativos, ya que se requiere estimular al estudiantado para despertar su curiosidad hacia lo que aprende con el uso de estas herramientas tecnológicas y de darle un nuevo enfoque al ambiente que se vive en nuestros salones de clase. Sin embargo, todavía existe personal educador reluctante al uso de los distintos dispositivos y herramientas que ofrece el maravilloso mundo de la tecnología, y el uso que el estudiantado les pueda dar a estos recursos en el aula, o en el aprendizaje de una lengua, como bien es el caso que aquí se presenta.

Una clase invertida de inglés utiliza las TIC para influenciar el ambiente de aprendizaje de quien se encuentra en un proceso de formación. En ella se proveen oportunidades para el aprendizaje y un mejor ambiente de aprendizaje para el grupo estudiantil con el uso de 
Figura 1. Modelo tradicional PPP.

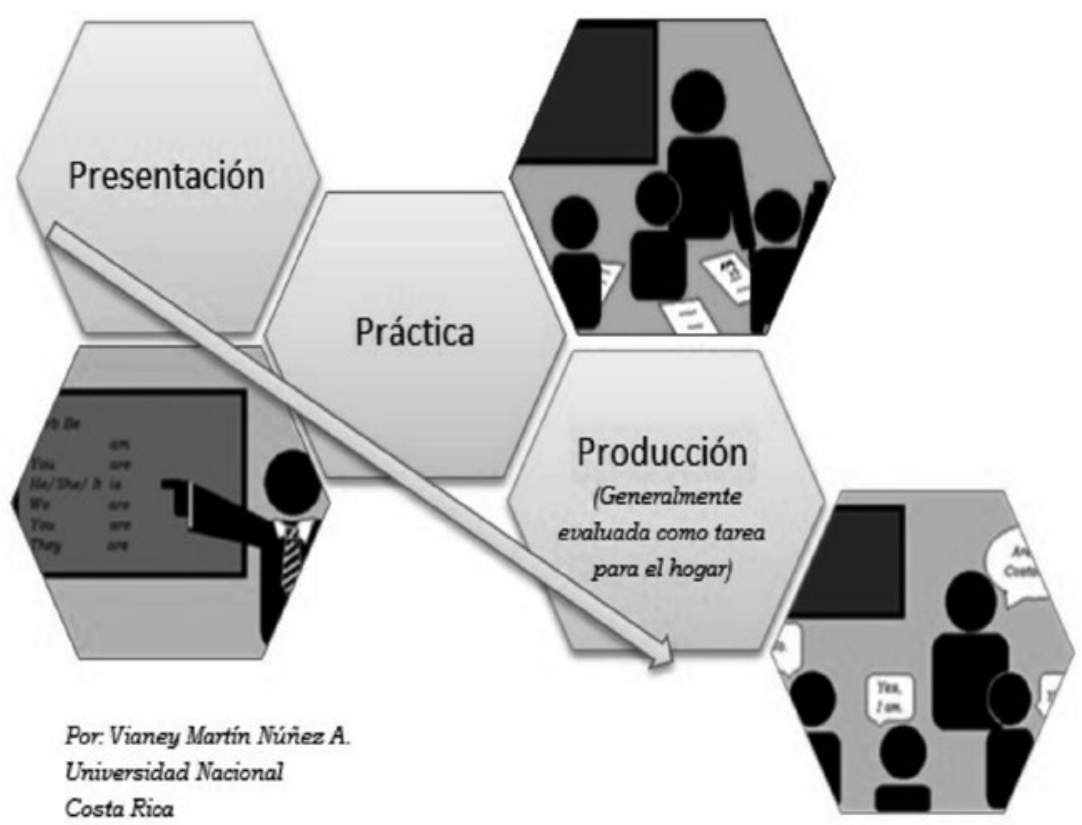

Fuente: Elaboración propia de Núñez, Martín.

múltiples aplicaciones y herramientas que permiten realizar actividades cuyo rango va desde lo básico hasta lo más complejo, y que desarrollan las diversas áreas lingüísticas desde la lectura hasta la expresión oral; incluyendo ejercicios como conversaciones, preguntas y respuestas, resolución de problemas, debates discusiones, juegos de rol o simulaciones, entre otros (Ver Figura 2).

\section{Contextualización}

Con el advenimiento de la globalización y la incorporación del país a distintos mercados mundiales, tanto el gobierno como la empresa privada han apuntado a fortalecer la enseñanza y aprendizaje del idioma inglés en los distintos niveles educativos. De este modo, desde el año 2008 las universidades estatales han venido incorporando una serie de medidas a nivel educativo que pretenden mejorar las competencias lingüísticas en el idioma inglés de la ciudadanía. 
La enseñanza invertida (EI) o flipped teaching (FT) como método de mediación pedagógica aplicado a un curso de Inglés Integrado para otras especialidades en la Universidad Nacional (UNA), Costa Rica

Figura 2. Modelo instrucción invertida (FT).

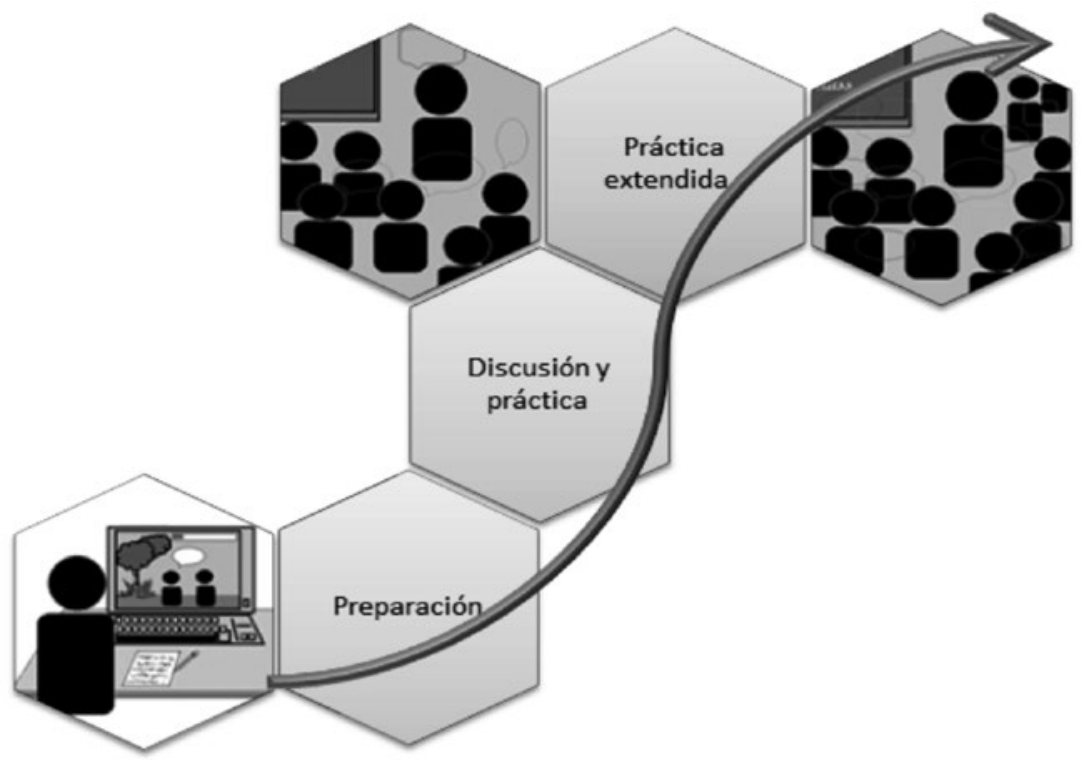

Fuente: Elaboración propia de Núñez, Martin (2015).

Según se indica en informes presentados a la Escuela de Literatura y Ciencias del Lenguaje por la Coordinación de Cursos de Servicios y la Coordinación Interinstitucional del Plan para el Fortalecimiento del Inglés en Universidades Públicas (PFI), una de estas medidas incluye la oferta de una serie de opciones formativas en lengua extranjera para estudiantado universitario. Esta oferta busca llevar a estudiantes desde el Nivel A1 al nivel B2 según el Marco Común Europeo de Referencia para las Lenguas Vivas, y en el caso particular de la Universidad Nacional se han incorporado a los diferentes planes de estudio de las distintas carreras ofertadas (Bolaños y Núñez, 2011; Núñez, 2015; Rodríguez, 2013).

El curso de Inglés Integrado II tiene como objetivo general desarrollar en el estudiantado las cuatro habilidades lingüísticas del idioma mediante diversas actividades de mediación pedagógica y se imparte cada semestre en ciclos que comprenden 8.1 horas de instrucción tanto presencial como de estudio independiente distribuidas en 17 semanas y que suman un total de 140 horas de instrucción. 
Así, la población base de este trabajo corresponde a estudiantes del curso Inglés Integrado II para Otras Carreras adscritos al Plan de Fortalecimiento del Inglés de la Escuela de Literatura y Ciencias del Lenguaje en la Universidad Nacional de Costa Rica (2008). La población que matricula este curso proviene de diversos contextos sociales, económicos, culturales y académicos, por lo que componen grupos extremadamente heterogéneos.

Esta diversidad en el estudiantado permea los procesos de enseñanza, aprendizaje y evaluación del inglés como lengua extranjera, pues resultan grupos igualmente diversos en cuanto a las competencias lingüísticas de sus componentes y con los desafíos propios que implican los grupos multinivel.

\section{Planteamiento de la investigación}

Por lo anterior descrito es que se considera importante desarrollar un estudio que permita dar respuesta a la siguiente pregunta de investigación:

¿Cuáles son las implicaciones de utilizar la metodología de la enseñanza invertida como instrumento de mediación pedagógica en los cursos de Inglés Integrado para otras Carreras II de la UNA?

\section{Objetivos generales y específicos}

Para contestar la pregunta anterior se ha planteado como objetivo general el evaluar los alcances de la utilización de la metodología de la enseñanza invertida como instrumento de mediación pedagógica en los cursos de inglés integrado para otras carreras en la UNA. Pero para alcanzar este objetivo, se plantean también como objetivos específicos el identificar las fortalezas y debilidades del alumnado en los cursos de inglés integrado II, el determinar y definir el nivel lingüístico de ingreso y de egreso del estudiantado.

\section{Tipo de investigación}

Sustentado en la metodología de la investigación cualitativa, este estudio se define como un estudio de caso el cual, según autores como Hernández, Fernández y Baptista (2014), corresponde al estudio descriptivo realizado a través de observaciones hechas por quienes investigan y 
están a cargo de impartir los cursos de Inglés Integrado II y de entrevistas informales a estudiantes, una vez realizadas las actividades de clases en las que se implementó el enfoque de la enseñanza invertida.

En cada momento en que se utilizó el enfoque de la enseñanza invertida en la clase de inglés se le asignó al estudiantado observar un video que se subió en un blog creado para este propósito, y que fue creado con la herramienta Web Blogger. En ella los estudiantes se encargaban de bajar el video, observarlo para que pudieran ser partícipes de una sesión de discusión que se realizaría en la clase siguiente con la guía de un grupo de preguntas que la profesora a cargo preparó con este objetivo. Además se incluyeron en este mismo blog algunos textos, fotos y otros con los cuales el estudiantado se ayudaba para reaccionar con sus opiniones en la clase, realizar más sesiones de discusión y escribir párrafos en donde incluyeran sus puntos de vista sobre los artículos leídos, fotos vistas, etc.

\section{Selección de la población y muestreo}

En este trabajo se seleccionó un grupo de 24 estudiantes del curso Inglés Integrado II para Otras Carreras a cargo de uno de los investigadores. Una selección que se realizó por conveniencia, y que radica en la cercanía y el fácil acceso que tendrían las personas a cargo del estudio a la información generada de la implementación del modelo de enseñanza invertida.

Es, de la misma manera, un artículo que abarca lo descriptivo, heurístico e inductivo al permitir la obtención e interpretación de información específica sobre la utilización de los recursos tecnológicos e informáticos como el internet, los videos, el blog y distintos medios sociales.

Una vez hechas las primeras observaciones y entrevistas realizadas por la docente a cargo, se determinó que las fortalezas más importantes de este alumnado radican en su deseo de mejorar el nivel lingüístico en idioma inglés que dicen traer del sistema de educación secundario. Adicionalmente, el grupo etario que conforma la población en estudio (18 a 23 años) permite la implementación de nuevas metodologías y estrategias de aprendizaje con apoyo de los recursos tecnológicos vigentes.

Por otro lado, las observaciones y entrevistas iniciales también demostraron que la mayor debilidad del alumnado es precisamente la multiplicidad de niveles con que cuenta. Gra cantidad de estudiantes 
tiene un conocimiento limitado del idioma meta, lo que interfiere con el desarrollo de la clase y la cobertura apropiada de contenidos léxicos, gramaticales y fonéticos en general.

Otra debilidad encontrada en el alumnado es que a pesar del interés demostrado en aprender un idioma extranjero, este es solamente un requisito de graduación en sus respectivas carreras; por lo tanto, se convierte no en una prioridad sino en una carga con la que deben cumplir y a la que generalmente no se le brinda el tiempo de estudio correspondiente.

\section{Recolección de los datos}

Con el fin de recolectar información viable y brindar validez a este trabajo investigativo, se elaboraron una lista de cotejo para las observaciones en aula, preguntas abiertas para la entrevista, dos cuestionarios dirigidos a estudiantes y un examen de diagnóstico inicial y final (ver apéndices).

En la tabla de cotejo o guía de observación se incluyeron descriptores relacionados con la motivación intrínseca y extrínseca, el uso del tiempo, el uso del lenguaje meta, las estrategias de aprendizaje empleados por el estudiantado, y las técnicas de enseñanza utilizadas por el profesorado (ver apéndice A).

Para la elaboración de la entrevista se incluyeron preguntas de respuesta abierta dirigidas a conocer aspectos meramente concernientes a las realidades lingüísticas y académicas del cuerpo estudiantil (ver apéndice B).

Adicionalmente, los cuestionarios buscaron determinar dos momentos distintos; uno inicial y uno final; en la implementación de la metodología de EI, resaltando aquellas ventajas, limitantes y alcances psico-sociales que tienen los entornos virtuales de educación en los procesos de auto-aprendizaje y su utilización o sub-utilización para el aprendizaje de una lengua extranjera (ver apéndices $\mathrm{C} \mathrm{y} \mathrm{D).}$

Además, para medir el nivel de lengua de ingreso y egreso, se aplicó en dos diferentes momentos de la investigación una prueba abreviada de dominio lingüístico (estos documentos debido a su extensión no son incluidos en este artículo) para medir algún cambio en el desempeño oral y escrito de la lengua meta. Así, el diagnóstico inicial se realizó en la semana número 1 del curso y el diagnóstico final se realizó en la semana 17. 


\section{Análisis de las categorías}

Una vez recolectada y tabulada la información, desde la tipología del estudio de caso, se procedió a comparar, contrastar, analizar y discutir sobre aquellos aspectos recurrentes, las tendencias sobresalientes, y los fenómenos psico-sociales derivados de la aplicación de la metodología EI que resultan particularmente interesantes para la investigación. Asimismo, no se descartan de ninguna manera las afirmaciones que puedan parecer opuestas por cuanto estas brindan, además, un rico ejercicio de análisis.

Una vez esquematizados y organizados los datos derivados, se procedió a establecer los criterios finales de la investigación, los cuales se exponen en el siguiente apartado a manera de conclusiones.

\section{Resultados y discusión}

El método de enseñanza invertida es una metodología innovadora que beneficia tanto al estudiantado como al profesorado. Esta aseveración se puede constatar y resumir con los resultados de lo observado y lo analizado.

Así, en primer lugar, relacionado con nuestros objetivos específicos se pueden desprender las siguientes conclusiones:

\section{a. Fortalezas del alumnado}

Una vez hechas las primeras observaciones y entrevistas se determinó, mediante el análisis de los resultados, que las fortalezas más importantes de este alumnado radican en su deseo de mejorar el nivel lingüístico en idioma inglés que dicen traer del sistema de educación secundario. Adicionalmente, el grupo etario que conforma la población en estudio (18 a 23 años) permite la implementación de nuevas metodologías y estrategias de aprendizaje apoyado en los recursos tecnológicos vigentes.

\section{b. Debilidades del alumnado}

Por otro lado, el análisis de las observaciones, entrevistas iniciales y la prueba diagnóstica de dominio lingüístico inicial, también 
demostró que la mayor debilidad del alumnado es precisamente la multiplicidad de niveles con que cuenta. La mayoría de estudiantes tiene un conocimiento limitado del idioma meta lo que interfiere con el desarrollo de la clase y la cobertura apropiada de contenidos léxicos, gramaticales y fonéticos en general.

Otra debilidad encontrada en el alumnado es que, a pesar del interés demostrado en aprender un idioma extranjero, este es solamente un requisito de graduación en sus respectivas carreras; por lo tanto, se convierte no en una prioridad sino en una carga con la que deben cumplir y a la que generalmente no se le brinda el tiempo de estudio correspondiente.

c. Nivel lingüístico de ingreso

Realizada la primera mediación mediante una entrevista oral y un examen de diagnóstico, se determinó que el nivel lingüístico en inglés de la población en estudio no es el apropiado para el curso. Así, en la figura 3 se demuestra la distribución porcentual según los niveles lingüísticos establecidos por el MCER:

Figura 3. Nivel de dominio lingǘ́stico inicial.

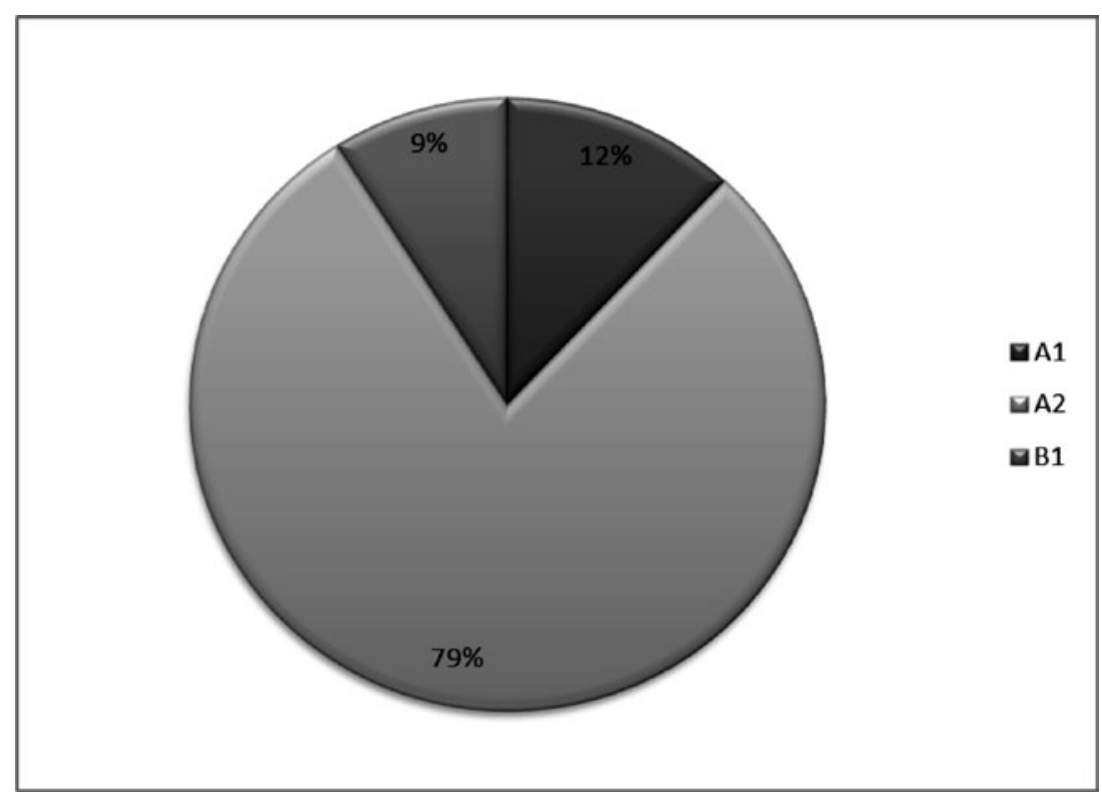

Fuente: Elaboración propia. 
La enseñanza invertida (EI) o flipped teaching (FT) como método de mediación pedagógica aplicado a un curso de Inglés Integrado para otras especialidades en la Universidad Nacional (UNA), Costa Rica

Como se desprende del gráfico anterior, el grueso de la población estudiantil ingresa al curso con un nivel A2, o principiante medio. Sin embargo, cerca del $12 \%$ de la población no tiene el conocimiento necesario en el idioma para incorporarse satisfactoriamente al curso. El manejo lingüístico de esta población es básico; en otras palabras, se manejan solamente términos aisladas o bien frases simples para brindar información personal. Adicionalmente, existe una población en el nivel B1, quienes pueden hacer uso de manera más fluida y eficiente del idioma; pero que, debido a circunstancias propias del sistema educativo universitario, deben o necesitan matricular el curso tal y cual se oferta.

d. Nivel lingüístico de egreso

Luego de 16 semanas de instrucción y la implementación de la metodología de la enseñanza invertida como método complementario al desarrollo de las lecciones se procedió a realizar una segunda medición y se obtuvo el siguiente resultado (Ver Figura 4).

Figura 4. Nivel de dominio lingüístico de egreso.

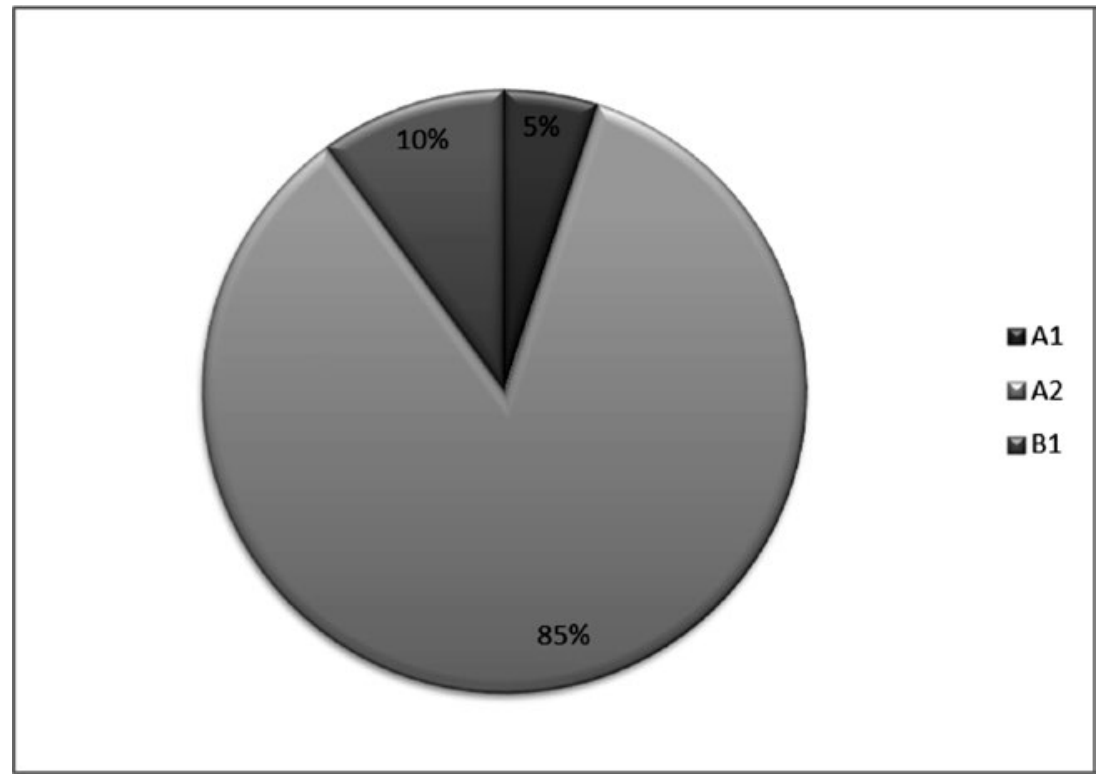

Fuente: Elaboración propia. 
Si se comparan ambos gráficos se puede desprender que más de la mitad de estudiantes que se encontraban inicialmente en el nivel A1 lograron dar el salto hacia el A2, mientras que la mayoría de estudiantes del nivel A2 lograron consolidarlo, y aunque de manera muy reservada al menos el uno por ciento del estudiantado logró avanzar hasta el nivel B1, lo cual implica una clara mejoría en el manejo integral del idioma meta.

\section{e. Limitantes}

En relación con las diversas limitantes que se pueden encontrar para la implementación de la metodología de la enseñanza invertida se destacan el acceso al recurso digital, el desconocimiento por parte de estudiantes y profesorado de ciertas competencias digitales, el tiempo que se invierte en la auto-instrucción, la creación de una cultura de auto-aprendizaje y el desarrollo de las habilidades organizativas por parte de quienes aprenden.

\section{f. Alcances psico-sociales}

La aplicación de la metodología enseñanza invertida o flipped teaching, en el Curso de Inglés Integrado para otras especialidades en la Universidad Nacional, Costa Rica, durante 210 horas lectivas distribuidas en 17 semanas de estudio se trató de buscar una alternativa a la clase tradicional que se ha venido implementado.

Para realizar este proceso, y con el fin de motivarlo y despertarle el interés hacia una nueva forma de aprender la lengua extranjera, se conversó con el estudiantado y se le explicaron los principios de una enseñanza invertida, los ejercicios en inglés que realizarían, los procedimientos que seguirían durante las lecciones de inglés, y el tiempo en que estarían trabajando con esta metodología innovadora.

Una vez que la docente a cargo consideró que el estudiantado había entendido las pautas a seguir, se empezó a trabajar con las actividades de mediación en donde se utilizaron algunas herramientas TIC y medios de comunicación social, tales como el uso de Blogger, videos en Youtube, Wikis, y Vokies, entre otros. Unas actividades que siempre fueron acompañadas con el uso de discusiones en clase, análisis 
de textos, conversaciones en grupos, y debates, entre otros, y que se desarrollaron durante las 17 semanas del ciclo lectivo.

Así, concluido este tiempo y además de realizar algunas observaciones mientras el alumnado participaba en las actividades seleccionadas, los responsables de esta investigación crearon el espacio en clase para conversar y así conocer los puntos de vista sobre la experiencia vivida.

\section{Conclusiones}

Como se ha discutido anteriormente, la metodología de la enseñanza invertida puede aportar algunas ventajas al aula de idioma como lengua extranjera; dentro de las ventajas demostradas en este grupo en particular se logró identificar, mediante el análisis de los resultados obtenidos en observaciones, entrevistas, cuestionarios, pruebas de diagnóstico y la evidencia de acceso a la página o aula virtual, el empoderamiento del propio aprendizaje, el fortalecimiento del pensamiento crítico, el desarrollo de espacios de formación autónoma, el reforzamiento de habilidades y competencias, la identificación de debilidades propias, el re-direccionamiento de la instrucción, y el apoyo individual y personalizado, y una interacción estudiante-docente y estudiante-estudiante más oportuna y eficaz.

Así mismo, y según las observaciones hechas, se pudo comprobar que en la mayoría del estudiantado participante en el estudio se fomentó el pensamiento crítico, y la resolución de problemas, mientras interactuaban utilizando la lengua meta, entre sí y con la profesora.

Por consiguiente, la EI resultó ser una metodología innovadora que promovió un ambiente diferente de clase, en donde el grupo de estudiantes participaba e interactuaba activamente y hacían uso de las experiencias, competencias y habilidades particulares de cada integrante para resolver las situaciones que se presentaban, encausar el nuevo conocimiento que adquirían y ser parte de su proceso de aprendizaje. 


\section{Referencias}

Bergmann, J. y Sams, A. (2012). Flip your Classroom: Reach Every Student in Every Class Every Day (en línea). Internacional Society for Technology in Education. Library of Congress. Recuperado de https://books.google.co.cr/books?hl=es\&l$\mathrm{r}=\& \mathrm{id}=-\mathrm{YOZCgAAQBAJ} \& \mathrm{oi}=$ fnd $\& \mathrm{pg}=$ PR 7\&dq=Flip+your+Classroom:+Reach+Every+Student + in $+\% 09$ Every++Class + Every+Day\&ots $=$ AEi9HMpsmg\&sig=F-M2Zuzym8unnBiabpPZ1XucuVI\&redir_esc $=\mathrm{y} \# \mathrm{v}=$ onepage $\& \mathrm{q}=\mathrm{Flip} \% 20$ your $\% 20$ Classroom $\% 3 \mathrm{~A} \% 20$ Reach $\% 20$ Every $\% 20$ Student $\% 20$ in $\% 20 \% 09$ Every $\% 20 \% 20$ Class $\% 20$ Every $\% 20$ Day\&f=false

Bolaños, I. y Núñez, M. (2011). Informe final del plan de fortalecimiento del Inglés. Coordinación Interinstitucional PFI, Cursos de Servicio. Universidad Nacional (UNA).

Boles, C. et al. (2014). The Flipped Classroom: An introduction to technology and teaching techniques (en línea). First Digital Printing. ISBN 978-1-63102-004-9. Recuperado de http://www.amazon. com/The-Flipped-Classroom-Introduction-Technology-ebook/ dp/B00K9N5X00

Bretzman, J. et al. (2013). Flipping 2.0-Practical Strategies for Flipping your class.Wisconsin:TheBretzmannGroup.Recuperadodehttp://www.amazon.com/The-Flipped-Classroom-Introduction-Technology-ebook/dp/ B00K9N5X00

EDUCAUSE. (2015). Learning Initiative. Things you should know about Flipped Classroom. Recuperado de http://net.educause. edu/ir/library/pdf/ELI7081.pdf. 1/30/2015

Hernández, R., Fernández, C y Baptista, P. (2014). Metodología de la Investigación ( $6^{\text {ta }}$ ed.). México, DF: McGraw Hill.

Murillo, J. et al. (2002) Estudio de casos [en linea]. Recuperado de https://www.uam.es/personal_pdi/stmaria/jmurillo/InvestigacionEE/Presentaciones/Curso_10/EstCasos_Trabajo.pdf

Núñez, M. (2015). Informe anual. Coordinación Interinstitucional PFI, Cursos de Servicio. Universidad Nacional (UNA).

Rodríguez, E. (2013). Informe parcial. Plan de fortalecimiento del inglés. Coordinación Interinstitucional PFI, Cursos de Servicio. Universidad Nacional (UNA). 
La enseñanza invertida (EI) o flipped teaching (FT) como método de mediación pedagógica aplicado a un curso de Inglés Integrado para otras especialidades en la Universidad Nacional (UNA), Costa Rica

Santiago, R. (2015). The Flipped Classroom [video]. Recuperado de $\mathrm{http}: / / \mathrm{www} \cdot$ theflippedclassroom.es/

Tourón. J. (2013). La enseñanza inversa y el desarrollo del talento. A propósito de manifiesto [en linea]. Recuperado de http://www.javiertouron.es/2013/09/la-ensenanza-inversa-y-el-desarrollo.html

Universidad Nacional. Escuela de Literatura y Ciencias del Lenguaje. (2008). Plan del fortalecimiento del Inglés de la Escuela de Literatura y Ciencias del Lenguaje en La Universidad Nacional e Costa Rica. 


\section{Apéndice A}

\section{Universidad Nacional}

Escuela de Literatura y Ciencias del Lenguaje Departamento de Inglés

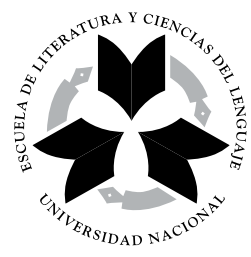

\section{Guía de observación}

\begin{tabular}{|l|l|l|}
\hline & Sí & No \\
\hline La profesora asignó un video / videos previa sesión. & & \\
\hline El estudiantado se muestra motivado por la asignación dada. & & \\
\hline $\begin{array}{l}\text { El estudiantado demuestra haber visto y analizado el video/videos } \\
\text { asignados }\end{array}$ & \\
\hline El estudiantado utiliza el idioma meta. & & \\
\hline El estudiantado hace preguntas o discute en relación con el video & & \\
\hline La profesora acompaña y monitorea la discusión generada. & & \\
\hline $\begin{array}{l}\text { El estudiantado demuestra haber comprendido la temática/contenidos } \\
\text { del video }\end{array}$ & \\
\hline $\begin{array}{l}\text { El estudiantado utiliza en su producción los contenidos estudiados } \\
\text { en el video }\end{array}$ & \\
\hline La profesora facilita la producción oral de sus estudiantes. & \\
\hline La profesora facilita la producción escrita de sus estudiantes. & \\
\hline $\begin{array}{l}\text { La profesora facilita la interacción estudiante-profesora, estudiante- } \\
\text { estudiante. }\end{array}$ & \\
\hline La interacción en clase evidencia el pensamiento crítico de estudiantes. & \\
\hline La interacción en clase evidencia la autonomía estudiantil. & \\
\hline $\begin{array}{l}\text { La interacción en clase evidencia la capacidad de solucionar } \\
\text { problemas del estudiantado. }\end{array}$ & \\
\hline El tiempo de producción extendida resulta suficiente. & \\
\hline El tiempo de producción extendida resulta eficiente. & \\
\hline El tiempodeproducción extendidaresulta agradableparael estudiantado. & & \\
\hline Comentarios adicionales & \\
\hline
\end{tabular}




\section{Apéndice B}

Guía de preguntas para el estudiantado

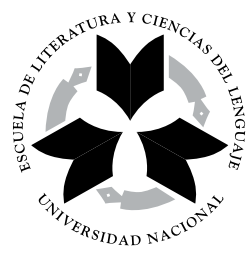

1. ¿Ha estudiado inglés anteriormente? ¿Dónde? ¿Por cuánto tiempo?

2. ¿Cuál ha sido su experiencia aprendiendo el idioma inglés? Explique.

3. ¿Qué nivel de inglés cree usted que tenga en el uso del idioma inglés: principiante, intermedio o avanzado?

4. ¿Cree usted que su nivel de inglés le permite comunicarse efectivamente con hablantes nativos del inglés? Explique

5. ¿Considera usted que su nivel de inglés le permite escuchar canciones y comprender videos o películas en el idioma inglés? Explique

6. ¿Considera usted que su nivel de inglés le permite leer textos en el idioma ingles y comprender su contenido? Explique

7. ¿Cree usted que su nivel de inglés le permite transmitir mensajes complejos o textos extensos en el idioma inglés? Explique

8. ¿Qué actividades recuerda usted han sido las más utilizadas por docentes de inglés en los cursos que ha tomado?

9. ¿Qué actividades recuerda usted que fueron poco implementadas pero que causaron algún impacto en su aprendizaje?

10. ¿Cuál ha sido la dinámica regular de las clases de inglés integrado en la UNA?

11. ¿Considera usted que una persona puede aprender el idioma inglés solamente a través de videos? Explique

12. ¿Considera usted que el profesorado de inglés utiliza demasiado 
tiempo en dar explicaciones gramaticales y poco tiempo para la práctica? Explique

13. ¿Considera usted que las actividades realizadas en el aula de inglés integrado ayudan al estudiantado a utilizar el idioma de forma práctica? Explique

14. ¿Considera usted que el estudiantado podría desarrollar mejores técnicas de aprendizaje del idioma si los recursos utilizados en el aula fuesen diferentes? Explique

15. ¿Cuál es su nivel de compromiso en el aprendizaje del idioma inglés? ¿Por qué?

16. Otras 


\section{Universidad Nacional}

\section{Apéndice C}

\section{Escuela de Literatura y Ciencias del Lenguaje Departamento de Inglés}

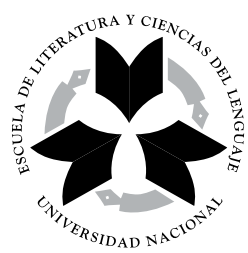

Estimados/as estudiantes:

Con el fin de brindar re-alimentación al proceso de enseñanza del inglés en los cursos de Inglés Integrado les solicitamos respetuosamente se sirvan contestar esta breve encuesta. La información aquí obtenida es de carácter confidencial y se utilizará para mejorar aquellas debilidades presentes y fortalecer nuestros aciertos.

Por favor conteste los siguientes enunciados marcando (ü) el espacio que más se acerque a su opinión sobre el aspecto planteado

\section{Edad}

$\begin{array}{ll}\square \text { menor de } 18 \text { años } & \square 18-23 \\ \square 30-35 & \square \text { mayor de } 35 \text { años }\end{array}$

2. ¿Cuál considera usted que es su nivel de inglés?

$\square$ Ninguno $\square$ Bajo $\square$ Intermedio $\square$ Alto

3. ¿Considera usted que deba mejorar su nivel de inglés? $\square \mathrm{Si} \square$ No ¿Por qué razón?

4. ¿Por qué razón matriculó el curso de inglés integrado? (puede marcar más de una opción)

$\square$ Requisito carrera
$\square$ Trabajo

$\square$ Interés personal
$\square$ Otro

5. ¿Cuál de los siguientes esquemas le resulta más común para el aula de inglés integrado?

$\square$ docente explica $\longrightarrow$ estudiante escucha $\longrightarrow$ docente dirige $\longrightarrow$ estudiante practica 
$\square$ docente explica $\longrightarrow$ estudiante escucha $\longrightarrow$ estudiante practica $\longrightarrow$ se asigna tarea

$\square$ estudiante práctica $\longrightarrow$ docente dirige $\longrightarrow$ estudiante practica $\rightarrow$ docente corrige

estudiante se prepara en casa $\longrightarrow$ docente dirige $\longrightarrow$ estudiante practica $\longrightarrow$ tarea

$\square$ estudiante se prepara en casa $\longrightarrow$ estudiante y docente practican $\longrightarrow$ estudiante produce

$\square$ Otro (grafique)

3. ¿Encuentra usted alguna ventaja o beneficio en ese diseño en particular?

$\square$ Sí $\square$ No

Explique

4. ¿Cuál de los siguientes esquemas le resulta menos común en el aula de inglés integrado?

$\square$ docente explica $\longrightarrow$ estudiante escucha $\longrightarrow$ docente dirige

$\longrightarrow$ estudiante practica

$\square$ docente explica $\longrightarrow$ estudiante escucha $\longrightarrow$

estudiante practica $\longrightarrow$ se asigna tarea

$\square$ estudiante hace $\longrightarrow$ docente dirige $\longrightarrow$ estudiante practica

$\longrightarrow$ docente corrige

$\square$ estudiante se prepara en casa $\longrightarrow$ docente dirige $\longrightarrow$ estudiante practica

$\square$ estudiante se prepara en casa $\longrightarrow$ estudiante y docente practican $\longrightarrow$ estudiante produce

5. De los siguientes enunciados, marque (ü) aquellos que son verdaderos según su experiencia en el aula de inglés integrado.

() El tiempo utilizado por el profesorado para explicar una temática es insuficiente.

El profesorado utiliza mucho tiempo de lección para explicar contenidos ( ) o temáticas. 
La enseñanza invertida (EI) o flipped teaching (FT) como método de mediación pedagógica aplicado a un curso de Inglés Integrado para otras especialidades en la Universidad Nacional (UNA), Costa Rica

( ) El profesorado utiliza videos y otros recursos para explicar contenidos o temáticas.

El profesorado sugiere videos y otros medios para ser consultados fuera

( ) del aula, según la temática del curso.

El profesorado asigna videos para ser analizados fuera del aula pero previo

( ) a cada sesión, según la temática del curso.

( ) El tiempo de práctica de una temática en clase por parte del estudiantado es insuficiente.

( ) El tiempo invertido en clase por el estudiantado en la práctica de una temática es suficiente

El estudiantado recurre, por su propia voluntad, al uso de videos y otros

( ) medios para mejorar su conocimiento en la temática del curso.

El estudiantado, por su propia voluntad, recurre al uso de videos y otros

( ) medios para mejorar su conocimiento en el idioma meta.

El estudiantado utiliza, si se le asigna, videos y otros medios para mejorar

( ) su conocimiento en la temática del curso.

El estudiantado utiliza, si se le asigna, videos y otros medios para mejorar

( ) su conocimiento en el idioma meta

( ) El tiempo invertido en clase para el uso y la producción del idioma meta es insuficiente.

( ) El tiempo invertido en clase para el uso y la producción del idioma meta es satisfactorio.

( ) Las clases de inglés integrado son entretenidas e innovadoras.

( ) Las clases de inglés permiten y potencializan el auto-aprendizaje del idioma meta. 


\section{Apéndice D \\ Universidad Nacional \\ Escuela de Literatura y Ciencias del Lenguaje \\ Departamento de Inglés}

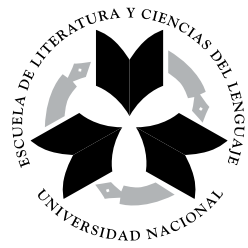

Estimados estudiantes:

Con el fin de brindar re-alimentación al proceso de enseñanza del inglés en los cursos de Inglés Integrado con la implementación del enfoque de la enseñanza invertida (FT) les solicitamos respetuosamente se sirvan contestar esta breve encuesta. La información aquí obtenida es de carácter confidencial y se utilizará para mejorar aquellas debilidades presentes y fortalecer nuestros aciertos.

Por favor conteste los siguientes enunciados marcando (ü) el espacio que más se acerque a su opinión sobre el aspecto planteado

1. ¿Cuál de los siguientes esquemas le resultó más evidente para el curso de inglés integrado?

$\square$ docente explica $\longrightarrow$ estudiante escucha $\longrightarrow$ docente dirige $\rightarrow$ estudiante practica

$\square$ docente explica $\longrightarrow$ estudiante escucha $\longrightarrow$ estudiante practica $\longrightarrow$ se asigna tarea

$\square$ estudiante práctica $\longrightarrow$ docente dirige $\longrightarrow$ estudiante practica $\longrightarrow$ docente corrige

$\square$ estudiante se prepara en casa $\longrightarrow$ docente dirige $\longrightarrow$ estudiante practica $\longrightarrow$ tarea

$\square$ estudiante se prepara en casa $\longrightarrow$ estudiante y docente practican $\longrightarrow$ estudiante produce

2. ¿Encontró usted alguna ventaja o beneficio en ese diseño en particular? Explique

$\square$ Sí $\square$ No 
La enseñanza invertida (EI) o flipped teaching (FT) como método de mediación pedagógica aplicado a un curso de Inglés Integrado para otras especialidades en la Universidad Nacional (UNA), Costa Rica

3. De los siguientes enunciados, marque (ü) aquellos que son verdaderos según su nueva experiencia en el aula de inglés integrado.

() El tiempo utilizado por el profesorado para explicar una temática es insuficiente.

() El profesorado utiliza mucho tiempo de lección para explicar contenidos o temáticas.

() El profesorado utiliza videos y otros recursos para explicar contenidos o temáticas.

( ) El profesorado sugiere videos y otros medios para ser consultados fuera del aula, según la temática del curso.

( ) El profesorado asigna videos para ser analizados fuera del aula pero previo a cada sesión, según la temática del curso.

() El tiempo de práctica de una temática en clase por parte del estudiantado es insuficiente.

() El tiempo invertido en clase por el estudiantado en la práctica de una temática es suficiente

( ) El estudiantado recurre, por su propia voluntad, al uso de videos y otros medios para mejorar su conocimiento en la temática del curso.

( ) El estudiantsdo, por su propia voluntad, recurre al uso de videos y otros medios para mejorar su conocimiento en el idioma meta.

( ) El estudiantado utiliza, si se le asigna, videos y otros medios para mejorar su conocimiento en la temática del curso.

( ) El estudiantado utiliza, si se le asigna, videos y otros medios para mejorar su conocimiento en el idioma meta

() El tiempo invertido en clase para el uso y la producción del idioma meta es insuficiente.

( ) El tiempo invertido en clase para el uso y la producción del idioma meta es satisfactorio.

( ) Las clases de inglés integrado son entretenidas e innovadoras.

( ) Las clases de inglés permiten y potencializan el auto-aprendizaje del idioma meta. 
4. ¿Con cuál de los siguientes enunciados está usted de acuerdo o no? Marque (ü) la columna correspondiente que mejor refleje su respuesta.

\begin{tabular}{|l|l|l|l|}
\hline & & & \\
& & & \\
\hline $\begin{array}{l}\text { La metodología implementada resultó interesante e } \\
\text { innovadora. }\end{array}$ & & & \\
\hline La metodología implementada resultó útil a mis intereses. & & & \\
\hline $\begin{array}{l}\text { La metodología implementada me permitió la auto- } \\
\text { formación en el idioma. }\end{array}$ & & & \\
\hline $\begin{array}{l}\text { La metodología implementada me permitió desarrollar el } \\
\text { pensamiento crítico. }\end{array}$ & & & \\
\hline $\begin{array}{l}\text { La metodología implementada me permitió empoderarme } \\
\text { de mis responsabilidades como estudiante. }\end{array}$ & & & \\
\hline $\begin{array}{l}\text { La metodología implementada me permitió un mayor uso } \\
\text { del idioma en el aula }\end{array}$ & & & \\
\hline $\begin{array}{l}\text { La metodología implementada me permitió un mejor uso } \\
\text { del idioma en el aula. }\end{array}$ & & & \\
\hline $\begin{array}{l}\text { La metodología implementada redujo el tiempo-cátedra del } \\
\text { personal docente en el aula }\end{array}$ & & & \\
\hline $\begin{array}{l}\text { La metodología implementada incrementó mi interacción } \\
\text { con otro estudiantado }\end{array}$ & & & \\
\hline Los videos asignados resultaron interesantes. & & \\
\hline $\begin{array}{l}\text { Los videos asignados resultaron útiles para el desarrollo de } \\
\text { la temática. }\end{array}$ & & & \\
\hline Los videos asignados resultaron de fácil comprensión. & & & \\
\hline $\begin{array}{l}\text { Los videos asignados redujeron mi tiempo de estudio para } \\
\text { otras materias. }\end{array}$ & & & \\
\hline La preparación previa sesión en el aula resultó demasiado larga. & & & \\
\hline $\begin{array}{l}\text { El tiempo brindado a la discusión del tema resultó demasiado } \\
\text { extenso. }\end{array}$ & & & \\
\hline $\begin{array}{l}\text { La realización de distintas actividades basadas en los videos } \\
\text { resultó difícil }\end{array}$ & & & \\
\hline Las distintas actividades basadas en los videos resultaron aburridas. & & & \\
\hline $\begin{array}{l}\text { Las distintas actividades basadas en los videos resultaron } \\
\text { fuera de contexto }\end{array}$ & & & \\
\hline
\end{tabular}

